\title{
Mixed Speculative Multithreaded Execution Models
}

\author{
POLYCHRONIS XEKALAKIS, NIKOLAS IOANNOU, and MARCELO CINTRA,
}

University of Edinburgh

The current trend toward multicore architectures has placed great pressure on programmers and compilers to generate thread-parallel programs. Improved execution performance can no longer be obtained via traditional single-thread instruction level parallelism (ILP), but, instead, via multithreaded execution. One notable technique that facilitates the extraction of parallel threads from sequential applications is thread-level speculation (TLS). This technique allows programmers/compilers to generate threads without checking for inter-thread data and control dependences, which are then transparently enforced by the hardware. Most prior work on TLS has concentrated on thread selection and mechanisms to efficiently support the main TLS operations, such as squashes, data versioning, and commits.

This article seeks to enhance TLS functionality by combining it with other speculative multithreaded execution models. The main idea is that TLS already requires extensive hardware support, which when slightly augmented can accommodate other speculative multithreaded techniques. Recognizing that for different applications, or even program phases, the application bottlenecks may be different, it is reasonable to assume that the more versatile a system is, the more efficiently it will be able to execute the given program.

Toward this direction, we first show that mixed execution models that combine TLS with Helper Threads (HT), RunAhead execution (RA) and MultiPath execution (MP) perform better than any of the models alone. Based on a simple model that we propose, we show that benefits come from being able to extract additional ILP without harming the TLP extracted by TLS. We then show that by combining all the execution models in a unified one that combines all these speculative multithreaded models, ILP can be further enhanced with only minimal additional cost in hardware.

Categories and Subject Descriptors: C.1.4 [Processor Architectures]: Parallel Architectures

General Terms: Design, Performance

Additional Key Words and Phrases: Speculative parallelization, speculative multithreading, runahead execution, multipath execution, helper threads

\section{ACM Reference Format:}

Xekalakis P., Ioannou N., and Cintra M. 2012. Mixed speculative multithreaded execution models. ACM Trans. Architec. Code Optim. 9, 3, Article 18 (September 2012), 26 pages.

DOI $=10.1145 / 2355585.2355591$ http://doi.acm.org/10.1145/2355585.2355591

\section{INTRODUCTION}

With the advent of multicore systems, the design effort has been alleviated from the hardware and placed instead on the compiler/programmer camp. Unfortunately, parallel programming is hard, and error-prone sequential programming is still

A preliminary version of this research was described in the International Conference on Supercomputing (ICS 2009) and in the International Conference on High Performance Computer Architecture (HPCA 2010). P. Xekalakis is currently affiliated with Intel Barcelona Research Center. N. Ioannou is currently affiliated with the IBM Zurich Research Lab.

Authors' addresses: P. Xekalakis, Intel-Labs Barcelona, C. Jordi Girona 9. 08034, Spain; email: polychronis. xekalakis@intel.com; N. Ioannou, IBM Zurich Research Lab, 8803 Rueschlikon, Switzerland; M. Cintra, University of Edinburgh, School of Informatics, 10 Crichton St. EH8 9AB, UK.

Permission to make digital or hard copies of part or all of this work for personal or classroom use is granted without fee provided that copies are not made or distributed for profit or commercial advantage and that copies show this notice on the first page or initial screen of a display along with the full citation. Copyrights for components of this work owned by others than ACM must be honored. Abstracting with credit is permitted. To copy otherwise, to republish, to post on servers, to redistribute to lists, or to use any component of this work in other works requires prior specific permission and/or a fee. Permissions may be requested from Publications Dept., ACM, Inc., 2 Penn Plaza, Suite 701, New York, NY 10121-0701 USA, fax +1 (212) 869-0481, or permissions@acm.org.

(c) 2012 ACM 1544-3566/2012/09-ART18 $\$ 15.00$

DOI 10.1145/2355585.2355591 http://doi.acm.org/10.1145/2355585.2355591 


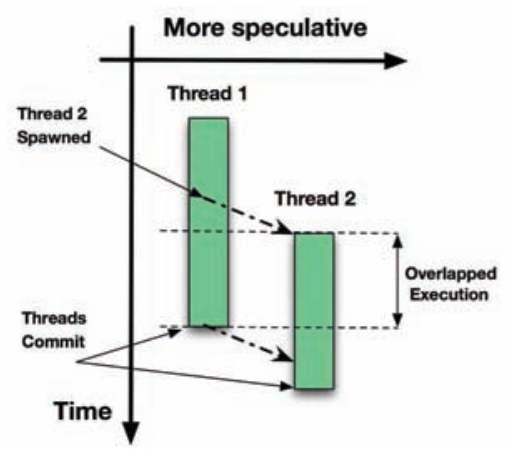

(a)

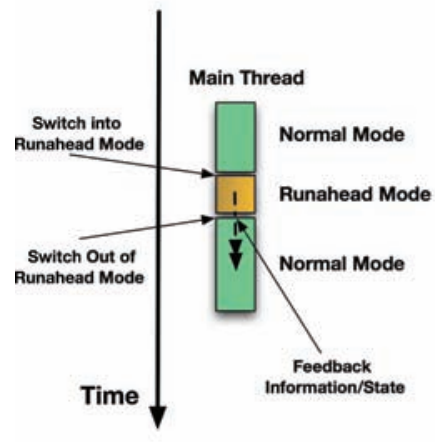

(c)

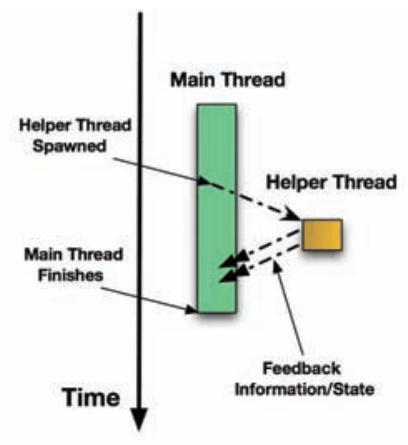

(b)

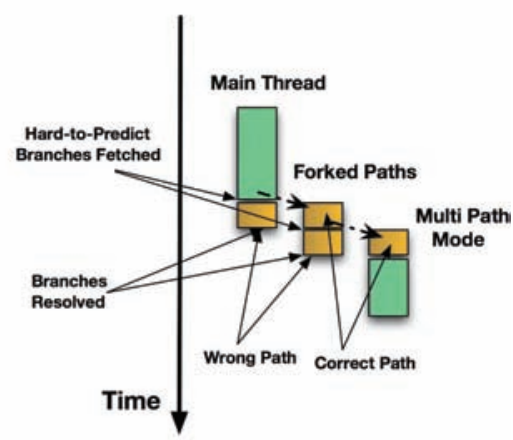

(d)

Fig. 1. Different models of speculative multithreaded execution: (a) Thread Level Speculation. (b) Helper Thread. (c) Runahead Execution. (d) Multipath Execution.

prevalent, and compilers still fail to automatically parallelize all but the most regular programs.

One possible solution to this problem is provided by systems that support ThreadLevel Speculation (TLS) [Hammond et al. 1998; Krishnan and Torrellas 1998; Marcuello and González 1999; Sohi et al. 1995; Steffan and Mowry 1998]. In these systems the compiler/programmer is free to generate threads without having to consider all possible cross-thread data dependences. Parallel execution of threads then proceeds speculatively and the TLS system guarantees the original sequential semantics of the program. Thus, the TLS model improves overall performance by exploiting Thread-Level Parallelism (TLP) via the concurrent execution of instructions in multiple threads ${ }^{1}$ (Figure 1(a)).

Another possible solution to accelerate program execution in multicore systems without resorting to parallel programs is provided by systems that support Helper Threads (HT) [Chappell et al. 1999; Collins et al. 2001; Sundaramoorthy et al. 2000; Zilles and Sohi 2001]. In these systems, the compiler/programmer extracts small threads, often called slices, from the main thread such that their execution in parallel with the main

\footnotetext{
${ }^{1}$ In reality, the basic TLS execution model also provides some indirect non-TLP performance benefits as explained later in the article.
} 
thread will lead to improved execution efficiency of the latter. Most commonly, HTs are used to resolve highly unpredictable branches and cache misses before these are required by the main thread. In almost all cases, the benefits of HT are indirect in the sense that no actual program computation is executed in parallel with the main thread. Thus, the HT model improves overall performance by exploiting InstructionLevel Parallelism (ILP) via the improved execution efficiency of a single main thread (Figure 1(b)).

A solution related to HT is to support RunAhead (RA) execution [Barnes et al. 2003; Ceze et al. 2006; Chaudhry et al. 2009; Dundas and Mudge 1997; Kirman et al. 2005; Mutlu et al. 2003]. In these systems, the hardware transparently continues execution of instructions from the main thread past a long latency operation, such as a cache miss. Unlike with HT, the instructions in the runahead thread are not explicitly extracted and placed in a subordinate thread and are not executed concurrently with the main thread. In fact, RA can be even implemented in single-core systems with some enhanced context checkpointing support. Like HT, the benefits from RA are indirect and it improves overall performance by exploiting ILP (Figure 1(c)).

Yet another possible solution to accelerate program execution without resorting to parallel programs is provided by systems that support Multi Path (MP) execution [Ahuja et al. 1998; Heil et al. 1996; Klauser et al. 1998]. In these systems, the hardware fetches along two paths for the hard-to-predict branches. The benefits of MP come from the fact that the processor is relieved from the misprediction penalty for the branches to which it is applied. Some benefits can also come from prefetching effects but typically they are not the main source of performance improvement over sequential execution. Thus, the MP model improves overall performance by exploiting ILP via the improved execution efficiency of a single main thread (Figure 1(d)).

Each of these four speculative multithreaded execution models has been separately shown to improve overall performance of some sequential applications. However, given the different nature of the performance benefits provided by each model, one would expect that combining them would lead to greater performance gains and over a wider range of applications compared to each model alone. Moreover, much of the architectural support required by each model is similar, namely: some support for checkpointing and/or multiple contexts, some support to maintain speculative (unsafe) data, and some support for squashing threads. Despite these opportunities no one (to the best of our knowledge) has attempted to combine these multithreaded execution models.

The main contribution of this article is to combine these four multithreaded execution models in a super-set model that can exploit the benefits of each model depending on application characteristics. More specifically, the resulting system attempts to exploit TLP speculatively with TLS execution, but when this fails or when additional opportunities exist for exploiting ILP the system also selectively employs a version of HT that is based on RA or MP execution. In the article we discuss in detail this interaction and how to tune the HT, RA, MP and TLS models to work synergystically. We use a simple methodology that allows one to model the performance gains with TLS and the mixed execution model such that gains can be accurately attributed to either TLP or ILP. Based on this, we reason about the behavior of the execution models and investigate tradeoffs in the mixed model. This article extends our prior published work by bringing together all four speculative multithreaded models. In Xekalakis et al. [2009] we mixed TLS with HT and RA and in Xekalakis and Cintra [2010] we mixed TLS with MP.

Experimental results show that the mixed execution model achieves speedups of up to $82 \%$, with an average of $14 \%$, over an existing state-of-the-art TLS system for a subset of the SPEC2000 Int benchmark suite. 
The rest of the article is organized as follows. Section 2 provides a brief description of the TLS, HT, RA and MP execution models. Section 3 presents the mixed TLS/HT/RA, TLS/MP and TLS/HT/RA/MP execution models. Section 4 presents our methodology for quantifying the contributions to performance gains that come from TLP and ILP. Section 5 describes the experimental methodology and Section 6 presents results. Finally, Section 7 discusses related work and Section 8 concludes the article.

\section{BACKGROUND}

\subsection{TLS}

Under the thread-level speculation (also called speculative parallelization or speculative multithreading) approach, sequential sections of code are speculatively executed in parallel hoping not to violate any sequential semantics [Hammond et al. 1998; Krishnan and Torrellas 1998; Marcuello and González 1999; Sohi et al. 1995; Steffan and Mowry 1998]. The control flow of the sequential code imposes a total order on the threads. At any time during execution, the earliest thread in program order is nonspeculative while the others are speculative. The terms predecessor and successor are used to relate threads in this total order. Stores from speculative threads generate unsafe versions of variables that are stored in some sort of speculative buffer. If a speculative thread overflows its speculative buffer it must stall and wait to become nonspeculative. Loads from speculative threads are provided with potentially incorrect versions. As execution proceeds, the system tracks memory references to identify any cross-thread data dependence violation. Any value read from a predecessor thread is called an exposed read, and it has to be tracked since it may expose a read-after-write (RAW) dependence. If a dependence violation is found, the offending thread must be squashed, along with its successors, thus reverting the state back to a safe position from which threads can be re-executed. When the execution of a nonspeculative thread completes it commits and the values it generated can be moved to safe storage (usually main memory or some shared lower-level cache). At this point its immediate successor acquires nonspeculative status and is allowed to commit. When a speculative thread completes it must wait for all predecessors to commit before it can commit. After committing, the core is free to start executing a new thread. Figure 1(a) illustrates this model.

Speculative threads are usually extracted from either loop iterations or function continuations. The compiler marks these structures with a forklike spawn instruction, so that the execution of such an instruction leads to a new speculative thread. The parent thread continues execution as normal, while the child thread is mapped to any available core/context. For loops, spawn points are placed at the beginning of the loop body, so that each iteration of the loop spawns the next iteration as a speculative thread. Threads formed from iterations of the same loop (and that, thus, have the same spawn point) are called sibling threads. For function calls, spawn points are placed just before the function call, so that the nonspeculative thread proceeds to the body of the function and a speculative thread is created from the function's continuation.

The architectural support required for any TLS system consists of six components: i) a mechanism to allow speculative threads to operate in their own context and to enforce that speculatively modified data be also kept separate, ii) a mechanism to track data accesses in order to detect any data dependence violations, iii) a mechanism to spawn threads in different cores or contexts, iv) a mechanism to rollback (i.e., squash and restart) incorrectly executed threads, v) a mechanism to commit the correctly speculatively modified data to the safe state, and vi) a mechanism to keep track of the ordering of threads with respect to the original sequential execution order. 


\subsection{HT}

Under the helper threads (also called subordinate microthreads) approach [Chappell et al. 1999; Collins et al. 2001; Sundaramoorthy et al. 2000; Zilles and Sohi 2001], small threads are run concurrently with a main thread. The purpose of the helper threads is not to directly contribute to the actual program computation, which is still performed in full by the main thread, but to facilitate the execution of the main thread indirectly. Common ways to accelerate the execution of the main thread involve initiating memory requests ahead of time (i.e., prefetching; such that the results are hopefully in the cache by the time they are needed by the main thread) and resolving branches ahead of time.

Usually, depending on how the helper threads are generated (see the following), the execution of helper threads is speculative in that they may be following some incorrect control flow path and/or producing and consuming incorrect data. In this speculative multithreaded execution model there is no particular ordering among multiple helper threads and all are discarded at the end of their execution. Figure 1(b) depicts this execution model.

Helper threads are usually generated by the compiler or programmer and often consist of slices of instructions from the main thread (e.g., only those instructions directly involved in the computation of some memory address or branch condition). Depending on the size and complexity of the helper threads it may be possible to keep all their intermediate results in the registers, but it may be necessary to allow for spills to the memory hierarchy, which in turn requires providing storage for speculative versions of data. The compiler marks the main thread with forklike spawn instructions at points where particular helper threads should be initiated.

The architectural support required by HT consists of three main components: i) a mechanism to allow helper threads to operate in their own context and, possibly, to enforce that speculatively modified data be also kept separate, ii) a mechanism to spawn threads in different cores or contexts, and iii) a mechanism to discard threads when finished.

\subsection{RA}

Under the runahead approach [Barnes et al. 2003; Ceze et al. 2006; Chaudhry et al. 2009; Dundas and Mudge 1997; Kirman et al. 2005; Mutlu et al. 2003], when the main thread hits a long-latency operation (e.g., an L2 miss) it halts execution and a runahead thread continues execution either ignoring or predicting the outcome of the long-latency operation. The purpose of the runahead thread is not to directly contribute to the actual program computation, which is often still performed in full by the main thread once it resumes, but to facilitate the execution of the main thread indirectly after it resumes. As with HT, common ways to accelerate the execution of the main thread involve prefetching and early branch resolution. Unlike HT, runahead threads do not run concurrently with the main thread. Thus runahead execution can be seen as a form of HTs which run only on idle cycles of the processor. The execution of the runahead thread is speculative since the outcome of the long-latency operation is either ignored or predicted. Thus, in most proposed models the runahead thread is discarded once the main thread resumes execution. In more aggressive models, however, if the predicted outcome of the long-latency operation is correct the execution of the runahead thread is incorporated into the main thread before stopping the execution of the runahead thread. Figure 1(c) shows this execution model.

Runahead threads are generated on-the-fly by the hardware and, like the common HT case, consist of a selection of instructions from the main thread. Strictly speaking, in many proposals in the literature, the runahead threads are in fact obtained by simply 
checkpointing the main thread and letting it run ahead instead of explicitly spawning a new thread elsewhere. Also like HT, it may be possible to keep all the intermediate results of the runahead thread in the registers, but it may be necessary to allow for spills to the memory hierarchy.

The architectural support required by RA consists of five main components: i) a mechanism to allow runahead threads to operate in their own context and, possibly, to enforce that speculatively modified data be also kept separate, ii) a mechanism to decide when to generate runahead threads or to switch the main thread into runahead mode, iii) a mechanism to discard incorrectly executed threads, and iv) and v) optional mechanisms to check if the runahead thread has executed based on correct or incorrect predicted outcomes and, if so, to incorporate the runahead state and data into the main thread.

\subsection{MP}

Under the multipath (MP) execution model [Ahuja et al. 1998; Heil et al. 1996; Klauser et al. 1998], both paths following a hard-to-predict conditional branch are followed. More specifically when a branch is thought ${ }^{2}$ to be a hard-to-predict one, another thread is created which is given a copy of the register file as it is before executing the branch. In order to allow fast register file copying, all threads are usually executed on the same core, which has multiple-context support. When the branch that triggered the MP execution is resolved, the thread that lies on the wrong path is discarded. Threads in MP mode cannot commit their state, since they might be executing instructions on the wrong path, thus intermediate stores are not propagated to the cache hierarchy - they are instead accommodated in the store buffers (in this model no spills are allowed). While executing in MP mode if there is no context available, subsequent hard-topredict conditional branches are typically treated as normal branches, that is, they are predicted using the branch predictor. MP is thus able to avoid branch mispredictions at the cost of executing more instructions. Figure 1(d) outlines this execution model.

The architectural support required by MP consists of three main components: i) a mechanism to allow MP threads to operate in their own context and, possibly, to enforce that speculatively modified data be also kept separate, ii) a mechanism to decide when to generate MP threads, and iii) a mechanism to discard incorrectly executed threads.

Table I summarizes the architectural support required by the four speculative multithreaded execution models in columns 2 to 5 (the last column shows the support used by our mixed TLS/HT/RA/MP scheme, which is described in Section 3).

\section{MIXED SPECULATIVE EXECUTION MODELS}

Each of the speculative multithreaded execution models that we consider-TLS, HT, $\mathrm{RA}$ and MP-is best at exploiting different opportunities for accelerating the performance of a single-threaded application. We thus expect a mixed scheme to perform as well as the best model across a variety of applications and even to outperform the best model. The latter can happen if the mixed scheme can adapt to the different acceleration opportunities of different program phases or if the acceleration opportunities are additive (e.g., if ILP can be exploited in addition to TLP for some program phase).

Since the hardware requirements of these execution models is similar, it is possible to tweak the execution models so that a system that supports one of them, can support the rest. In prior work we have crafted two such models, namely one that combines TLS with HT and RA execution [Xekalakis et al. 2009] and a more elaborate one that combines TLS with MP execution [Xekalakis and Cintra 2010].

\footnotetext{
${ }^{2}$ Confidence estimators are typically used to predict whether the branch predictor usually fails to predict correctly the specific branch.
} 
Table I.

Hardware support required for the different models of speculative multithreaded execution and for our mixed approach. $O$ stands for optional feature, $X$ stands for feature not required, and $\checkmark$ stands for feature required.

\begin{tabular}{lccccc}
\hline Mechanism & TLS & HT & RA & MP & Mixed \\
\hline Data Versioning & $\checkmark$ & $\checkmark$ & $\checkmark$ & X & $\checkmark$ \\
Data Dep. Tracking & $\checkmark$ & X & X & X & $\checkmark$ \\
Spawn Threads & $\checkmark$ & $\checkmark$ & X & $\checkmark$ & $\checkmark$ \\
Discard/Rollback & $\checkmark$ & $\checkmark$ & $\checkmark$ & $\checkmark$ & $\checkmark$ \\
Commit State & $\checkmark$ & X & O & X & $\checkmark$ \\
Order Threads & $\checkmark$ & X & X & X & $\checkmark$ \\
Checkpoint Threads & O & X & $\checkmark$ & X & $\checkmark$ \\
Value Predict L2 Misses & X & X & O & X & $\checkmark$ \\
\hline
\end{tabular}

In the next sections, we will start by describing the TLS/HT/RA execution model since it is the simplest and requires only minimal changes in the protocol of TLS. We will then proceed to present the TLS/MP execution model which is slightly more complex. We will then present a new model that combines all four simple execution models, requiring only minimal additions to the TLS/MP model.

\subsection{Combining TLS, HT, and RA Execution}

The basic idea of this mixed model is to start with TLS execution and to convert some TLS threads into a new type of thread which we term Prefetch Threads (PT). PTs are essentially TLS threads which go into runahead mode (RA) and try to prefetch for the rest of the TLS threads. Threads that have been converted to prefetch threads execute the same instructions as they would in TLS mode, but they are allowed to predict the results of L2 misses. Not having to stall, PTs are able to make forward progress much faster than normal TLS threads. These converted prefetch threads can no longer contribute to the actual parallel computation (i.e., they can never commit) but can only help the remaining TLS threads execute more efficiently by prefetching for them in the shared L2 cache. In a multicore environment with TLS this can be achieved when the converted prefetch threads bring data into L2 that will be later used by the remaining TLS threads. Note that in TLS the L1 cache is versioned so that no sharing and, thus, no prefetching, can occur across prefetch threads and TLS threads. PTs resemble HTs in that they both try to prefetch for other threads. However, they are different in how they are generated and how they manage to be ahead of the main thread(s). HTs are typically manually crafted and rely on profile information. PTs are dynamically constructed and rely on runtime information. HTs manage to be ahead in terms of execution from the rest of the threads by being spawned early and by containing only the backward slice of the problematic instructions (e.g., L2 cache misses). PTs on the other hand rely on runahead execution.

Combining TLS and PT is a reasonable approach for three main reasons. Firstly, because we start by employing only TLS, we first try to extract all the available TLP. This makes sense in a system with several cores since, if TLP can be exploited, it is more likely that this will yield better performance than trying to speed up a single main thread. When we fail to speculatively extract TLP, we may utilize the extra hardware resources to improve the ILP of the main thread, whereas in a base TLS system these resources would be idle. Secondly, accommodating HT and RA execution within the TLS execution model requires only slight modifications to the base TLS system. Finally, starting from TLS threads and implicitly generating runahead slices 
according to the RA model is a simple and automatic way of generating prefetch threads (no programmer/compiler intervention is required).

While the basic idea is simple, developing a fully working system requires dealing with a few implementation issues. The key issue relates to the policy of when, where and how to create PT. These decisions are critical because in our PT model, threads do not contribute to TLP and consume TLP resources (e.g., cores, caches), so that a conversion policy must balance the potential increase in ILP with the potential loss of TLP. Another aspect of this is whether prefetch threads are simply converted from existing TLS threads in place, or whether new TLS threads are specifically created elsewhere for the purpose of becoming prefetch threads. This decision also affects how to manage prefetch threads in the context of an extended TLS environment. In particular, the TLS environment imposes a total ordering on the threads in the system, which is reasonable for TLS threads, but becomes slightly more involved when some threads are TLS and some are PT. Also, a question is how to deal with a prefetch thread when it detects a data dependence violation and when one of its predecessors is squashed. Finally, another important issue relates to the policy of converting threads back from PT to TLS threads. Since our simplified PT model does not allow for their execution to be integrated into the TLS execution, this latter policy boils down to how/when to destroy PTs and free up resources for other TLS threads.

Figure 2 depicts how this mixed model operates. The only difference with the TLS execution model is that the most speculative thread is allowed to spawn PTs. More specifically, if the most speculative thread suffers a miss in the L2 cache and there is an available core in the system, a clone of this thread is spawned by it. This new thread is semantically the same as the spawner thread. However, the clone thread value predicts all misses in the L2 cache that it encounters and when it reaches the commit instruction (or causes an exception) it always gets killed. The clone thread has to also be killed if the spawnee thread encounters a spawn instruction, so as to preserve the rule that only the most speculative thread can have a PT. This constraint forces the clone thread to always be the most speculative and as such when it is killed there are no implications for the rest of the TLS threads. This constraint also means that a prefetch thread will never cause the squash of a TLS thread as the prefetch thread is always in the most-speculative position.

Although this model is fairly simple, it is able to significantly speed up the base TLS execution. Because all threads share a common level of cache (i.e., L2), there is no need to create multiple prefetch threads. In fact in Xekalakis et al. [2009] it was shown that this only provides marginal benefits. The main reason for this is that most of the L2 cache misses are first seen by the most speculative thread that executes code that would have been executed in the future by the sequential system (e.g., next iterations of a loop). This also suggests that the timeliness of these prefetches is also better.

\subsection{Combining TLS and MP Execution}

The next execution model we consider is the one that combines TLS with MP. MP has been studied for wide single core out-of-order systems, where it was reported to be able to save many pipeline flushes, albeit at a high cost in additional instructions executed. We note however, that MP execution makes even more sense for smaller cores, like the ones we use in our multicore, than it does for larger ones. MP execution is often considered a rather wasteful approach to remove costly branch mispredictions, since we typically have to execute a significant amount of extra instructions. For the simpler cores normally used to exploit TLP in TLS systems, however, due to their shallow pipelines, this is not the case since branches are resolved relatively fast. Small resolution times also suggest that one should be able to employ MP execution to a higher number of branches, since it is less probable that we will encounter too many 


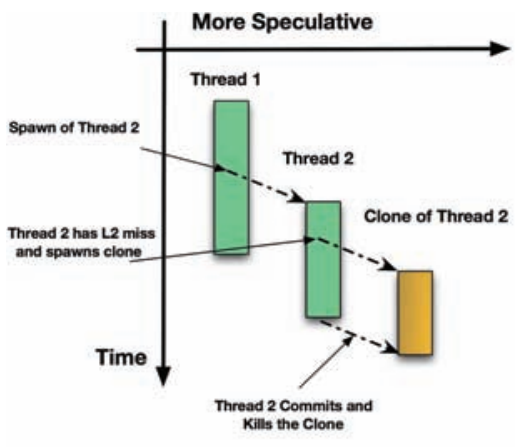

(a)

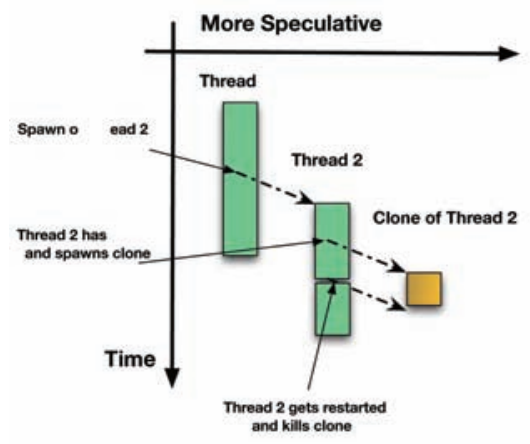

(c)

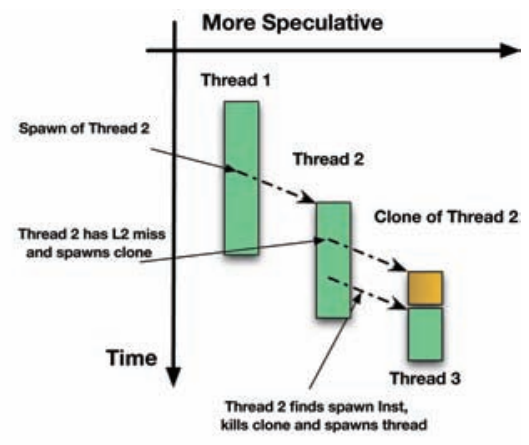

(b)

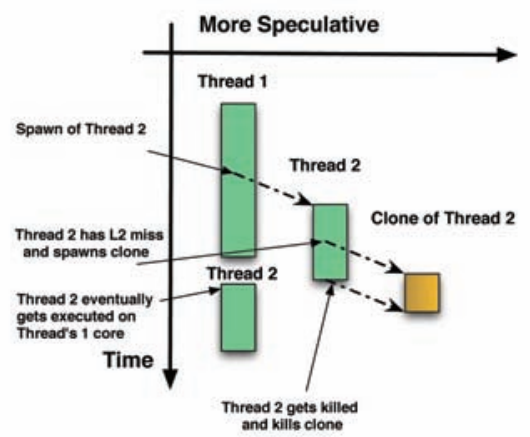

(d)

Fig. 2. Prefetch threading with clones: (a) A thread is cloned on an L2 miss. (b) The clone is killed on a thread spawn. (c) The clone is killed on a restart/kill of the thread that spawned it. (d) A TLS thread killed can execute on the same core as the parent thread if the parent thread has finished execution.

branch mispredictions before we resolve the previous ones. Note that although for sequential execution, smaller resolution times result in reduced performance benefits, this is not the case for TLS, where mispredictions cost more than they do for sequential execution. This increased cost is a consequence of the wider execution window that is exposed by TLS (thus mispredictions flush larger number of instructions).

In order to be able to support MP execution efficiently, we need to enhance our cores with multiple-context execution. The reason for this is that under MP execution we have to be able to perform fast register file copying when we decide to follow alternate paths. We will further discuss how this affects our system later in this section.

Figure 3 depicts this mixed execution model. Under this scheme there are two operational modes, normal TLS and MP mode (we only show four paths for clarity). We enter MP mode when we find a hard-to-predict branch and if there is a free context available on the core running the thread. When the branch is resolved, we follow the correct path and discard the incorrect one. When all pending hard-to-predict branches are resolved we exit MP mode.

In order to support the additional operations we keep some extra information which has to do with the path each of the threads in MP mode is following and how many outstanding paths we currently have. We thus, hold per thread an extra bit, the $M P$ bit, which indicates whether the thread runs on MP mode or not. To keep track of the outstanding paths we require per task an additional three bit counter (we allow six paths). We call the bits of this counter the PATHS bits, and we increment them 
every time we create a MP thread and we decrement each time we kill a MP thread. So far, the two threads that follow alternate paths are identical. In order to be able to differentiate between them, we keep per task an additional three bits, the DIR bits, which indicate the direction that the thread has followed since it started in MP mode (i.e., taken or not-taken). Since while executing in MP mode, we are not sure which of the two threads will be the one that will be kept and which will be discarded, we have to treat them as if they were the same thread in terms of the version of data they read. At the same time we must be able to differentiate which exposed reads have been performed by which thread so that we do not unnecessarily kill any thread because its wrong path clone performed a violating access. Since we keep information about exposed reads at the cache lines, we have to augment them with the DIR bits as well. When two threads that follow alternate paths perform a read, they do not need to consult their DIR bits (i.e., they only use the version ID as normal TLS threads). However, when they keep their own copy of the cache line, instead of only tagging it with the version ID, they also use the DIR bits. In this way when a read is found to be violating, we can check whether it was performed on the wrong or the correct path, and restart only if necessary. When a subsequent thread wishes to read a value, it may read it without consulting these extra bits. Note that because under MP mode all the stores are kept in the store buffers until the branch commits, control-speculative values are not propagated to the versioned memory and are, thus, transparent to the TLS protocol. Thus, threads that run on MP mode, cannot cause squashes to other threads. When a branch that triggered MP execution is resolved, the PATHS counter is decreased by one, and the DIR bits of the two threads are checked accordingly.

If a predecessor thread performs a store that violates a load in a thread running on MP-mode, we do not restart the thread immediately. We instead wait until all the branches are resolved and we take action then accordingly (in a fashion similar to delayed disambiguation schemes).

As Figure 3(a) shows, normal TLS threads have zeroes to all these extra bits. When a low confidence branch is encountered (Figure 3(b)), the thread executing follows the predicted path, while another thread is spawned that follows the alternate path. Both threads set their MP bit to 1, indicating they run in MP mode, update the PATHS counter and consult it so as to set the DIR bit accordingly (if PATHS is 1, the first DIR bit should be changed). When a second low confidence branch is encountered (Figure 3(c)), the PATH counter is updated (the MP is already set for the two pre-existing threads so it only needs to be set on the newly created thread). Note that now the spawnee thread cannot follow any path as it would have to update all of its cache lines, it thus follows the path that will leave its DIR bits the same (in this case the '000' path). Once the first branch is resolved, the thread that was executing on the wrong path is discarded and the remaining threads decrement their PATHS counter by one. Once the PATHS counter is zero again, it means that the TLS thread now operates in normal TLS mode.

Finally, two implications have to be dealt with: the first is that we have so far silently assumed that the branches will be resolved in the order they created the paths. However, since this may not be true, we must have a way to either prevent it from happening or keep track of which branch causes the creation of which thread. We choose the first, since the ROB already provides the required support. More specifically, we do not inform the system that the MP branches are resolved if all the previous MP branches in the ROB have not yet been resolved. As such we can be sure that the correct ordering is maintained. The second implication is how we handle spawn instructions while in MP mode (so that threads are not created on the wrong path). In order to prevent the spawning of threads while on the wrong path, we pessimistically suppress 


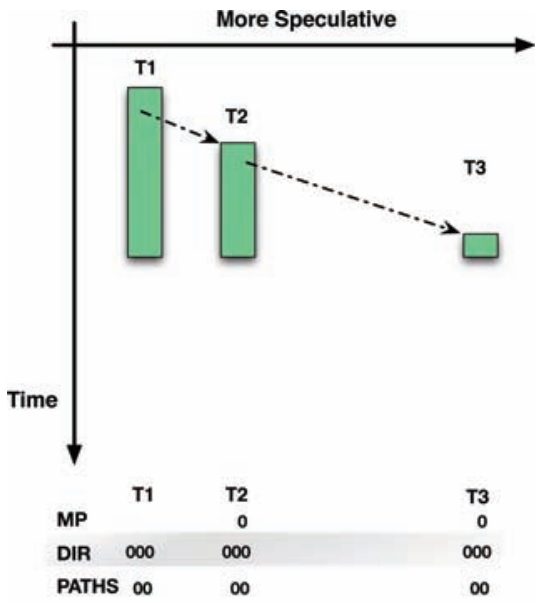

(a)

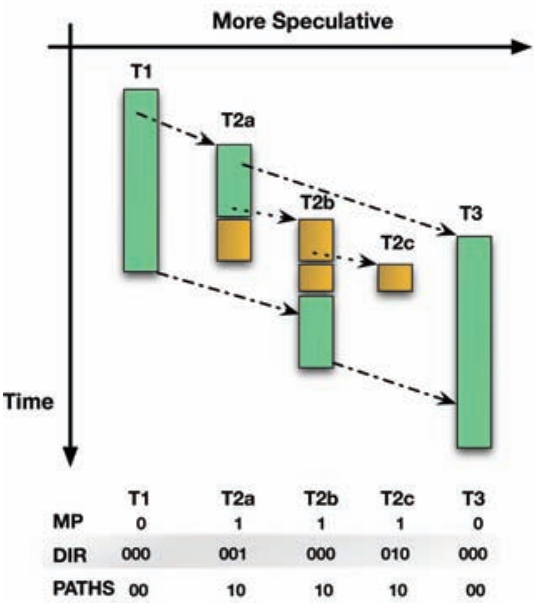

(c)

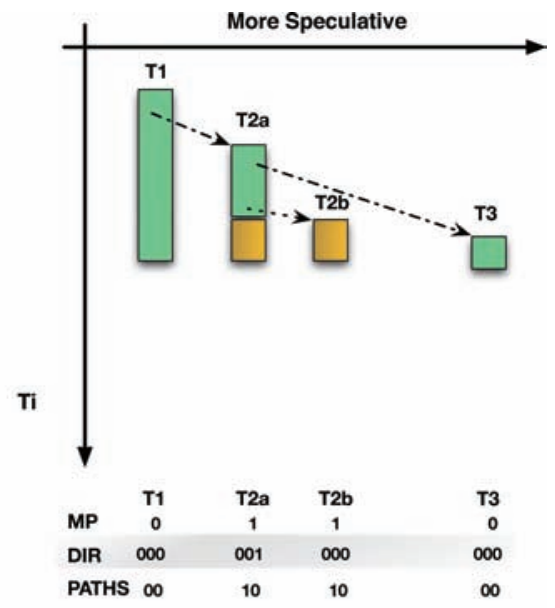

(b)

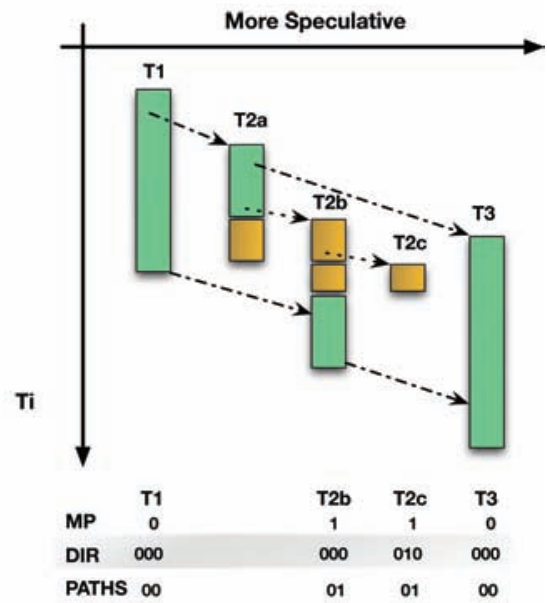

(d)

Fig. 3. Mixed TLS/MP scheme along with the additional MP, DIR and PATH bits: (a) Normal TLS spawns threads when it encounters spawn instructions. (b) On a "hard-to-predict" branch in T2a we create a clone of the thread that follows the alternate path. MP and PATH bits are set to one and DIR bits are set accordingly. (c) A second "hard-to-predict" branch is encountered in T2b. The MP bit of the new thread is set to one, the PATH bits are incremented and the DIR bits are set so that they do not change the DIR bits of the spawnee thread. (d) The first "hard-to-predict" branch in T2a is resolved, we discard the thread that was on the wrong path (T2a) and continue execution. The PATH counter is also decremented.

them, in our effort to make the MP-execution as transparent as possible to the normal TLS execution.

Of course in order to create the thread running on the alternate path, we need fast register file copying (or fast copying of the rename tables). For this reason we rely on cores with multithreaded execution support [Tullsen et al. 1996]. MP threads will have to be created and mapped on the same core. Note that live-in passing from spawner to spawnee thread for these threads is not performed via memory as it is the case for normal TLS threads, since the compiler is not aware of their existence. 


\subsection{Putting it All Together}

Although the two previously described execution models both try to improve the ILP of TLS, they do so in a complementary fashion. The first achieves this goal by removing some of the costly accesses to main memory, while the latter does so by reducing the cost of branch mispredictions. Following the same line of thought, these two models may be mixed in one that encapsulates both.

Figure 4 outlines the operation of this new model. When a normal TLS thread encounters a miss in the L2 cache, a PT thread is spawned as it would have been in the TLS/HT/RA model. The HT bit of this newly created thread is set to one, while the rest of the bits, namely the MP, DIR and PATHS are left at 0 (Figure 4(a)). The HT/RA is mapped to a free core so as to leave space within the multiple-context core to spawn MP threads if this is needed. Although this results in slightly delaying the generation of the PT, this is acceptable in practice. In fact, since the PTs are generated on a miss in the L2, the time that they have at their disposal in order to provide some benefit due to prefetching is defined by the distance between two consecutive misses in the L2.

As with the TLS/HT/RA model, when a normal TLS thread encounters a spawn instruction, it issues a kill signal to the PT if there is one, and the new TLS thread is spawned (Figure 4(b)). As with the PT thread case, we choose to spawn TLS threads to a different core instead of doing so within the core. Again the main motivation behind this choice is that MP threads can only be spawned within the core, so that we wish to make room for them. One difference with the previous case (i.e., the HT/RA one) is that TLS threads are more sensitive to this additional delay [Warg 2005]. However, we have found that it is typically better to sacrifice some of the TLS benefit in order to attain additional benefits from the MP threads.

When hard-to-predict branches are encountered by TLS threads, the execution model is exactly the same as the TLS/MP one (Figure 4(c)). As with the previous models, threads in MP or PT mode do not spawn new TLS threads. Additionally, PT threads do not spawn MP threads. Although this would speed up the PT in reality the PT is already quite fast when compared to the rest of the threads, so that the extra cost of using more contexts does not pay off.

However, MP threads may spawn HT/RA threads (Figure 4(d)). This design decision leads to the question of what should be done for PT threads that were spawned from the wrong path. Killing this thread would result in very short-lived PTs, which would probably not have enough time to perform any useful prefetches. As mentioned earlier, the PTs require some time so as to be able to provide any meaningful benefits. For this reason, in this model, the PTs are only killed if there is a TLS thread spawn or if the PT reaches a commit instruction. Interestingly, we found that even though the PT may lie on the wrong path it may still be of use for two reasons: i) it prefetches for control independent code, and ii) branches that are hard-to-predict are typically branches whose outcome changes frequently. As such, the PT may prefetch for subsequent TLS threads (i.e., spawned from the next iterations of loops) and still be beneficial.

\section{QUANTIFYING PERFORMANCE GAINS IN SPECULATIVE MULTITHREADED EXECUTIONS}

With speculative multithreaded execution models part of the performance variation observed is due to overlapped execution of instructions from multiple threads where these instructions contribute to the overall computation; we call this a TLP contribution. Another part of the performance variation is due to indirect contributions that improve (or degrade) the efficiency of execution of the threads; we call this an ILP contribution. For instance, with TLS the parallel execution of threads leads to a TLP contribution but also prefetching effects may lead to an ILP contribution. This may 


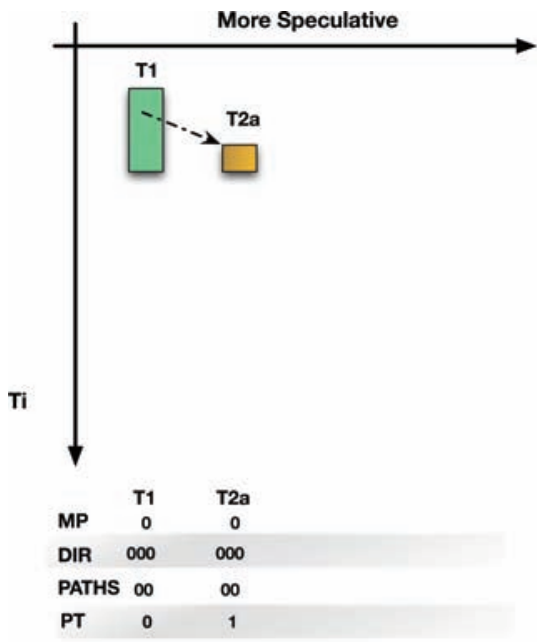

(a)

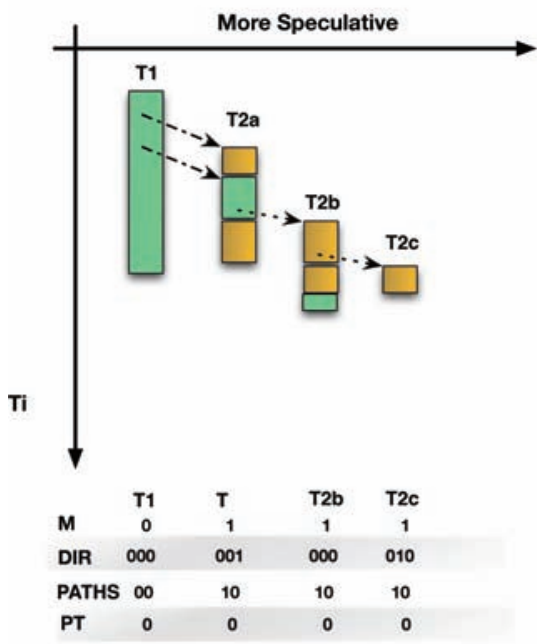

(c)

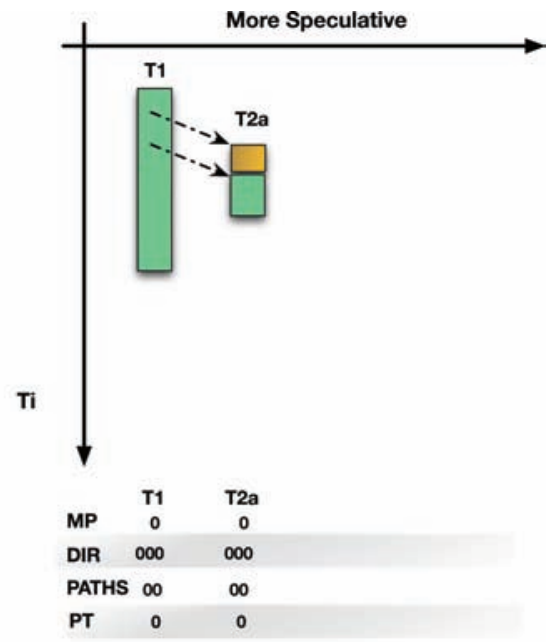

(b)

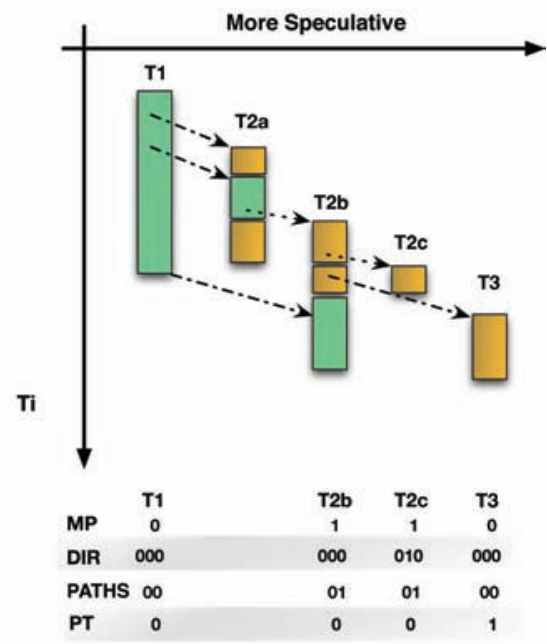

(d)

Fig. 4. Mixed TLS/MP/HT/RA scheme along with the additional MP, DIR, PATH and HT bits: (a) Normal TLS spawns a PT when it encounters a miss in the L2. The PT bit is set to one for the PT thread. (b) When a spawn instruction is encountered in a TLS thread, the PT is killed and the new thread is spawned. (c) "Hard-to-predict" branches are encountered resulting in the spawning of T2b and T2c. The MP, DIR and PATHS bits are set as they did for the TLS/MP model. (d) An L2 miss is encountered in T2b, which triggers the spawning of PT thread T3. T3 has all bits but the PT one set to 0. Although T2c is spawned this does not kill T3 since it is a MP thread.

happen when some threads share data so that the first thread to incur a cache miss effectively prefetches for the others such that the others will appear to have an improved ILP. Note that it is also possible that due to contention for resources, threads appear to have a degraded ILP. Note also that with speculative multithreaded models the possibility of squashes and re-execution of threads leads to even more intricate relationships between TLP and ILP contributions. Accurately quantifying the TLP and ILP contributions toward the final observed performance variation is critical in order 

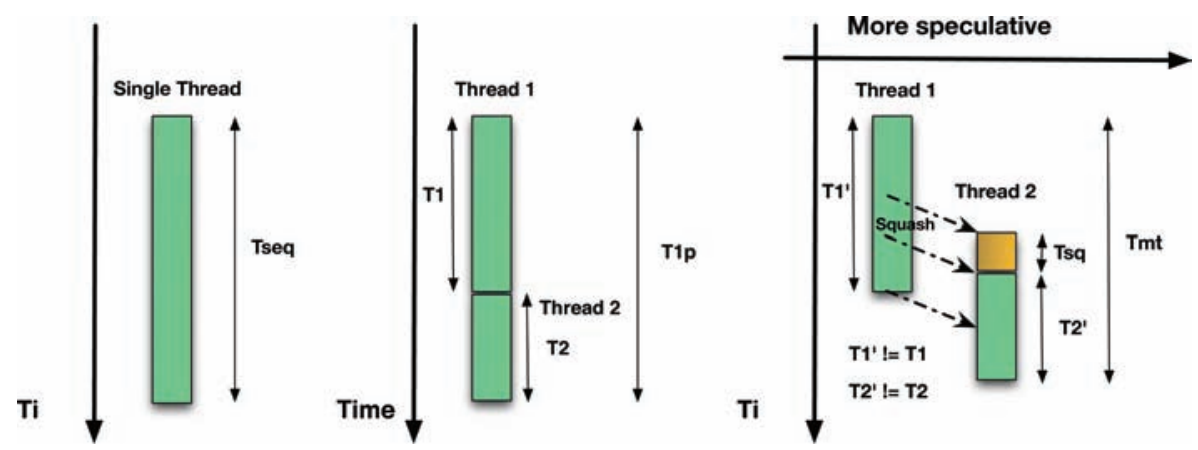

Fig. 5. Quantifying ILP and TLP benefits.

to reason and act upon the behavior of speculative multithreaded execution models. In this section we present a methodology to quantify these TLP and ILP contributions in speculative multithreaded execution models using easy to collect timing information from the actual execution. The model is a variation of that proposed in Renau et al. [2005]: it requires one extra simulation run but provides a more accurate estimate of the ILP contribution.

The performance model is based on measuring the following quantities from the execution: i) the execution time of the original sequential code $\left(T_{\text {seq }}\right)$; ii) the execution time of the modified TLS code when executed in a single core $\left(T_{1 p}\right)$; iii) the sum of execution times among all threads that actually commit $\left(\sum T_{i}\right.$, for all threads $i$ that commit); and iv) the execution time of the modified TLS code when executed in multiple cores $\left(T_{m t}\right)$. Figure 5 depicts these quantities for a simple example with two threads. With these quantities, the overall performance variation $\left(S_{\text {all }}\right)$ is given by Equation (1) and the performance variation (usually a slowdown) due to the TLS instrumentation overhead $\left(S_{1 p}\right)$ is given by Equation (2). The latter is needed in order to account for the variations needed in the binaries that execute the sequential and the multithreaded versions of the program.

$$
\begin{aligned}
S_{a l l} & =\frac{T_{s e q}}{T_{m t}} \\
S_{1 p} & =\frac{T_{s e q}}{T_{1 p}}
\end{aligned}
$$

The overall performance variation of the multithreaded execution over the TLS code executed in a single core $\left(S_{c o m b}\right)$ is given by Equation (3). This performance variation reflects the combined effects of both ILP and TLP and the equality shown in Equation (4) holds.

$$
\begin{gathered}
S_{c o m b}=\frac{T_{1 p}}{T_{m t}} \\
S_{c o m b}=S_{i l p} \times S_{t l p} .
\end{gathered}
$$

The performance variation of the multithreaded execution over the TLS code executed in a single core and that can be attributed to ILP effects $\left(S_{i l p}\right)$ is given by Equation (5).

$$
S_{i l p}=\frac{T_{1 p}}{\sum T_{i}}
$$


Thus, $S_{i l p}$ can be computed with the measurements of $T_{1 p}$ and all $T_{i}$ s. The performance variation of the multithreaded execution over the TLS code executed in a single core and that can be attributed to TLP effects $\left(S_{t l p}\right)$ can be computed by substituting the results of Equations (3) and (5) into Equation (4). The reason we compute $S_{t l p}$ indirectly is that directly measuring overlap, especially in the presence of task squashes and re-executions, is not straightforward and can lead to misleading results. Finally, we observe that the equality shown in Equation (6) holds, which shows that the final observed performance variation can be quantitatively attributed to the variations in the binary, to the ILP contributions, and to the TLP contributions.

$$
S_{a l l}=S_{1 p} \times S_{i l p} \times S_{t l p} .
$$

Comparing our model with that proposed in Renau et al. [2005] it can be shown that the key difference is that the ILP estimate in that model is ultimately derived from the "dynamic code bloat" factor $f_{\text {bloat }}$, while it is derived in our model from the actual instrumentation slowdown $S_{1 p}$. The problem with using $f_{\text {bloat }}$ as a proxy for the actual slowdown is that it implicitly assumes that the CPI of the unmodified sequential execution and that of the TLS-instrumented sequential execution are the same. In reality, however, the TLS instrumentation does affect the CPI as it involves the addition of mainly function calls and memory operations to set up thread spawns, which in turn have a different CPI from the rest of the thread. Obviously, this impact on CPI will be more pronounced for smaller threads than for larger ones.

\section{EVALUATION METHODOLOGY}

\subsection{Simulation Environment}

We conduct our experiments using the SESC simulator [Renau et al. 2004]. The main microarchitectural features are listed in Table II. The system we simulate is a multicore with 4 cores, where each core is 4 -issue out-of-order superscalar with support for 6 thread contexts. For the TLS protocol we assume out-of-order spawning [Renau et al. 2005]. The branch predictor on which we base our study is a state-of-the-art OGEHL predictor [Seznec 2005], which can also be ahead-pipelined. The minimum branch misprediction latency in our system is 16 cycles while we also employ speculative updates of the global history register.

In addition to the base architecture we require a confidence estimator and some extra bits in the cache. The confidence estimator is an important mechanism for MP execution, because it is used to trigger the MP execution mode. We use a 12-Kbit enhanced JRS confidence estimator [Grunwald et al. 2011] which uses 12-bits of prediction history. Using CACTI [Tarjan et al. 2006] we found that the overhead of the extra L1 bits needed to store the extra DIR bits was small enough, so as not to affect the number of cycles needed to access the L1.

\subsection{Benchmarks}

We use the integer programs from the SPEC CPU 2000 benchmark suite running the Reference data set. We focus on these applications because they represent a set of well accepted benchmarks that make difficult both the extraction of ILP (for MP and PT) and TLP (for TLS). We use the entire suite except eon, which cannot be compiled because our infrastructure does not support $\mathrm{C}++$, and gcc and perlbmk, which failed to compile in our infrastructure. For reference, the sequential (non-TLS) binaries where obtained with unmodified code compiled with the MIPSPro SGI compiler at the O3 optimization level. The TLS binaries were obtained with the POSH infrastructure [Liu et al. 2006]. We simulate enough simulation marks so that the corresponding sequential application graduates more than 750 million instructions. 
Table II.

Microarchitectural parameters of the simulated system along with the extra bits over the base TLS.

\begin{tabular}{ll}
\hline Parameter & \multicolumn{1}{c}{ TLS (4 cores) } \\
\hline Core Frequency & $3 \mathrm{GHz}$ \\
Contexts & 6 \\
Fetch/Issue/Retire Width & $4,4,5$ \\
L1 DCache/ICache & $32 \mathrm{~KB}, 4$-way, 3cycles/2 cycles \\
Main Memory & 300 cycles \\
I-Window/ROB & 40,100 \\
Ld/St Queue & 20,20 \\
Branch Predictor & $32 \mathrm{Kbit}$ OGEHL \\
BTB/RAS & $1 \mathrm{~K}$ entries, 2-way, 32 entries \\
Minimum Misprediction & 16 cycles \\
Cycles from Violation to Kill/Restart & 20 \\
Cycles to Spawn Same/Different Core & $1 / 20$ \\
\hline \multicolumn{2}{c}{ Additional Hardware } \\
\hline Confidence Estimator & $4 \mathrm{~K}$ Entries / 3bits Enhanced JSR \\
Extra Counters TLS/HT/RA & $(1 \times 1$ bit $) /$ Context \\
Extra Counters TLS/MP & $(1 \times 1$ bit, $1 \times 3$ bits, 1 x 2bits ) / Context \\
Extra Counters TLS/HT/RA/MP & $(1 \times 2$ bit, 1 x 3bits, 1 x 2bits ) / Context \\
\hline
\end{tabular}

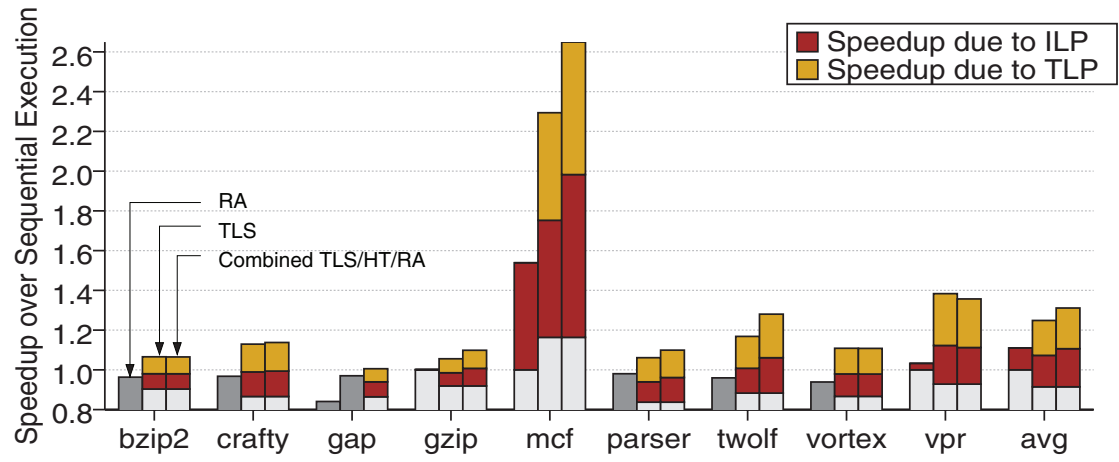

Fig. 6. Speedup of RA execution, a 4 core TLS system and the combined TLS/HT/RA scheme over sequential execution.

We employ the performance model discussed earlier (Section 4) throughout our evaluation section so as to accurately quantify the TLP and ILP impact on performance.

\section{EXPERIMENTAL RESULTS}

\subsection{Performance of Mixed Execution Models}

Figure 6 depicts the performance of RA execution, TLS execution, and the combined TLS/HT/RA scheme. With the light grey shade we plot the baseline relative performance from which the corresponding scheme starts. For the TLS based schemes this is usually below 1.0 (i.e, sequential) due to code bloat; (this is the $S_{1 p}$ factor of Section 4). The orange shade shows the proportion of TLP contribution to the total speedup, while 


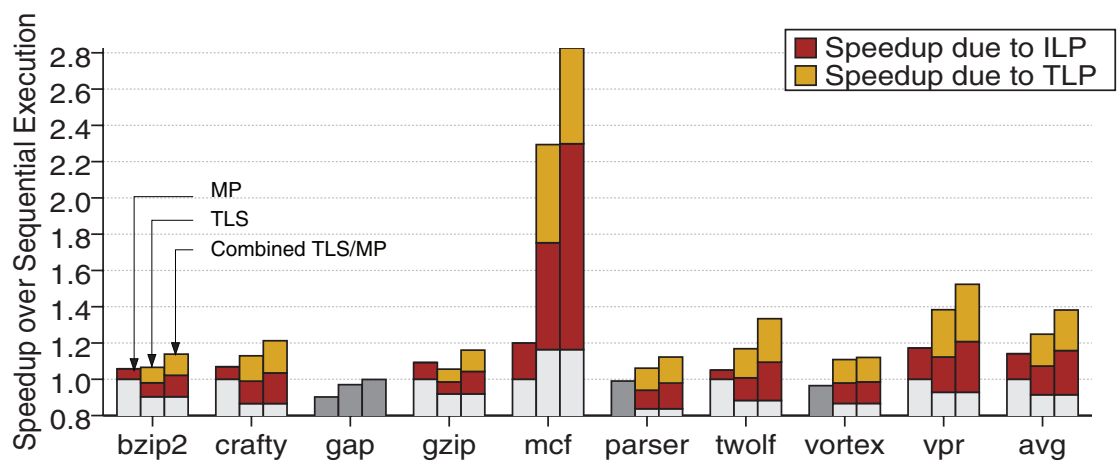

Fig. 7. Speedup of MP execution, a 4 core TLS system and the combined TLS/MP scheme over sequential execution.

the red shade shows the ILP contribution. ${ }^{3}$ For some of the benchmarks some of the schemes witness a slowdown: in these cases we use a darker grey shade to denote this.

Considering the base execution models alone, we first note that while TLS performs better than RA for most applications, for some applications the performance of both schemes is comparable (gzip and parser). We also note that, despite the main target of TLS being the exploitation of TLP, for most applications the benefits of ILP in the base TLS scheme are comparable to the TLP benefits.

Comparing the TLS/HT/RA scheme with TLS and RA, we see that the combined scheme performs at least as well as the best of other schemes for all applications and often outperforms the best scheme. The performance advantage of the combined scheme indicates that often the speedups of RA and TLS are additive. In fact, even in applications where RA fails to deliver any speedups (crafty, gap and vortex), the combined TLS/HT/RA scheme achieves speedups equal to or better than TLS. When compared to TLS, we see that the TLS/HT/RA scheme obtains greater performance gains from ILP while maintaining most of the TLP benefits. Interestingly, in some cases TLS/HT/RA is better than the base TLS scheme even in terms of TLP. One possible reason for this is that faster speculative threads will uncover violating reads faster and thus perform restarts earlier. This is an effect similar to having lazy or eager invalidations, where it is known that eager invalidations procure better results due to increased TLP. When compared to RA, we see that TLS/HT/RA obtains gains from TLP while maintaining most of the ILP benefits. Again, in some cases our unified scheme leads to higher ILP benefits than the base RA. The reason for this is the deeper prefetching that can be achieved by performing runahead execution from a speculative thread, which can be very far ahead from the main thread due to the way threads are created for TLS. Overall, we see that this simple mixed execution model achieves speedups of up to $41.2 \%$, with an average of $10.2 \%$, over the existing state-of-the-art TLS system and speedups of up to $35.2 \%$, with an average of $18.3 \%$, over RA with value prediction.

Figure 7 presents the performance results for the second mixed execution model (i.e., TLS/MP), when compared to MP execution and the base TLS. Note that MP execution can only get significant benefits over sequential execution for crafty $(5.2 \%), m c f(20.2 \%)$ and $\operatorname{vpr}(17.5 \%)$. Note also that for gap, parser and vortex it is actually slower than the sequential execution. This stems from the inability of the confidence estimator to accurately find branches that would have been mispredicted, which leads to unnecessary

\footnotetext{
${ }^{3}$ Note that the breakdown shows proportions of speedup due to each category and the height of each portion cannot be directly read as the speedup coming from ILP and TLP.
} 
Table III.

IPC of the sequential system, along with MPKI for the L2 cache and the branch predictor, the PVN (i.e., the probability a prediction estimated to have low confidence is incorrect), PVP (i.e., the probability a prediction estimated to have a high confidence is correct), SPEC (i.e., the probability that the confidence estimator reports an incorrectly predicted branch as having low confidence) and SENS (i.e., the probability that the confidence estimator reports a correctly predicted branch as a high confidence one).

\begin{tabular}{lcccccccccc}
\hline Statistic (\%) & Bzip2 & Crafty & Gap & Gzip & Mcf & Parser & Twolf & Vortex & Vpr & \multicolumn{1}{c}{ Avg. } \\
\hline Base IPC & 1.32 & 1.19 & 1.13 & 1.00 & 0.10 & 0.76 & 0.53 & 1.19 & 0.93 & 0.91 \\
L2 MPKI & 0.10 & 0.04 & 0.75 & 0.18 & 64.50 & 1.11 & 2.95 & 0.14 & 0.72 & 7.83 \\
Bpred MPKI & 5.62 & 4.01 & 0.99 & 5.24 & 8.86 & 3.97 & 14.45 & 0.42 & 8.07 & 5.73 \\
PVN & 22.8 & 16.9 & 19.5 & 24.1 & 27.9 & 20.8 & 23.2 & 11.6 & 24.4 & 21.3 \\
PVP & 98.2 & 97.6 & 98.8 & 98.6 & 99.2 & 98.9 & 96.4 & 99.8 & 98.0 & 98.4 \\
SPEC & 90.7 & 89.1 & 89.7 & 91.4 & 91.8 & 90.0 & 91.3 & 88.5 & 91.0 & 90.4 \\
SENS & 95.0 & 96.0 & 97.5 & 95.4 & 96.6 & 97.3 & 89.5 & 99.8 & 93.9 & 95.7 \\
\hline
\end{tabular}

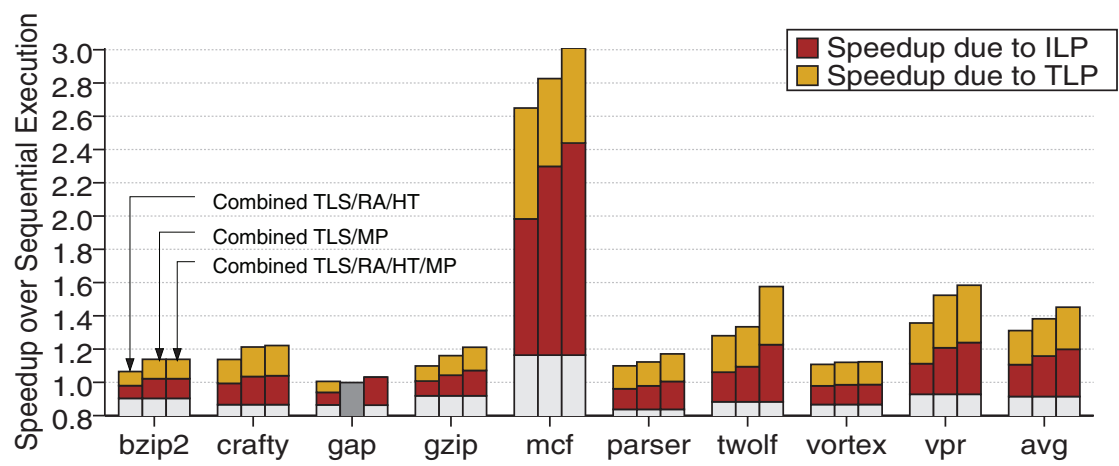

Fig. 8. Speedup of the combined TLS/HT/RA, the combined TLS/MP and the combined TLS/HT/RA/MP schemes over sequential execution.

increases in contention for functional units in the core. Despite the slowdown in these three applications, MP execution still manages to improve performance by $1.9 \%$ on average, a result which is in line with those previously reported in Kim et al. 2006].

The combined scheme is able to perform significantly better than both MP execution and the base TLS. More specifically, the combined scheme improves performance over TLS by as much as $23.2 \%$ ( $m c f$ ), with an average of $9.2 \%$. The only case where our combined scheme does not get any significant improvement over TLS is vortex. However, the misprediction rate for vortex is just $0.3 \%$ (Table III) and, as such, there is little room for improvement. Perhaps more importantly, for this application the use of MP reduces the amount of TLP that can be extracted using the base TLS, a fact that negates the improvements in ILP. It is interesting to note that with the combined scheme all of the benchmarks improve in terms of ILP, while at the same time they do not lose a significant amount of TLP. When we compare our scheme with MP execution, the achieved speedups are even more pronounced (28.2\% on average). Overall the combined execution model seems to enjoy an additive effect in terms of performance and manages to gain speedups over the sequential execution due to benefits arising from both improved ILP and extracted TLP.

Figure 8 shows the performance of the TLS/HT/RA/MP execution model, when compared with TLS/HT/RA and TLS/MP. For the memory bound $m c f$, the addition of PT threads leads to an increase in the ILP that can be extracted. Prefetching also helps $v p r$ and gzip, although it does so to a lesser degree. Perhaps the most interesting application for this combined scheme is twolf. This benchmark not only benefits from 


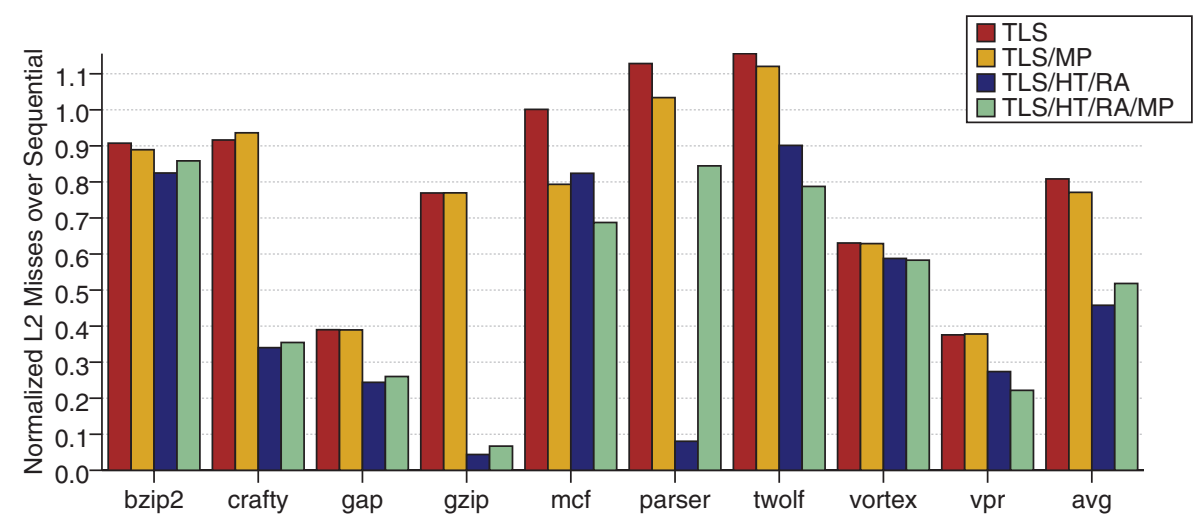

Fig. 9. Misses in the L2 cache for the TLS system and the combined TLS/MP, TLS/HT/RA, and TLS/HT/RA/MP schemes normalized over those of sequential execution.

prefetching when we add PT to the TLS/MP model (evident by the increase in ILP), but also sees TLP benefits. Investigating twolf deeper, we found that prefetching mainly affects the main thread, making it faster and increasing the amount of overlap with the more speculative threads. Overall, this new model is $15 \%$ better than the TLS/HT/RA model and $10 \%$ better than the TLS/MP one, while it is never worse than any of the two.

\subsection{Microarchitectural Analysis}

Table III summarizes the IPC, misses per thousand instructions for the L2 cache and the mispredictions per thousand of instructions for the baseline core. In the same table we also present the characterization of the confidence estimator for the base MP configuration. For the mixed execution models that include the MP execution model (i.e., TLS/MP and TLS/HT/RA/MP), these numbers are very similar. Along the lines of prior work on MP, the confidence estimator is biased to predicting branches as low confidence in order to increase coverage. Note that the mixed execution models improve performance mainly because of prefetching to the L2 and by removing some of the pipeline flushes due to mispredictions (most of the benefits come in terms of an increase in ILP).

Figure 9 presents, normalized over sequential execution, the number of L2 misses for TLS and the three combined models. Note that for the speculative models, we only account for misses that occur in the commit path, because these are the ones that directly contribute to ILP. As the graph reveals, even for the base TLS there is a substantial prefetching effect. Interestingly this trend is also true for the TLS/MP model, and in fact it is even slightly more pronounced because speculative threads are faster and tend to prefetch more. ${ }^{4}$ As expected the models that target prefetching more directly enjoy a substantial reduction in the number of misses. Of course, since the TLS/HT/RA/MP model shares the resources with the MP threads, it loses some of the prefetching benefits. However, it is able to close the gap between TLS/MP and TLS/HT/RA, and in the cases of $m c f$, twolf and $v p r$ even be slightly better.

At the same time the TLS/HT/RA/MP model is able to remove many of the flushes due to branch mispredictions. This is shown in Figure 10, where the number of flushes that are removed thanks to the ability to go along multiple paths is shown, for the MP,

\footnotetext{
${ }^{4}$ This is not necessarily due to wrong path prefetching. Note that even if TLS/MP threads are on the correct path, they still can be squashed due to data dependence violations.
} 


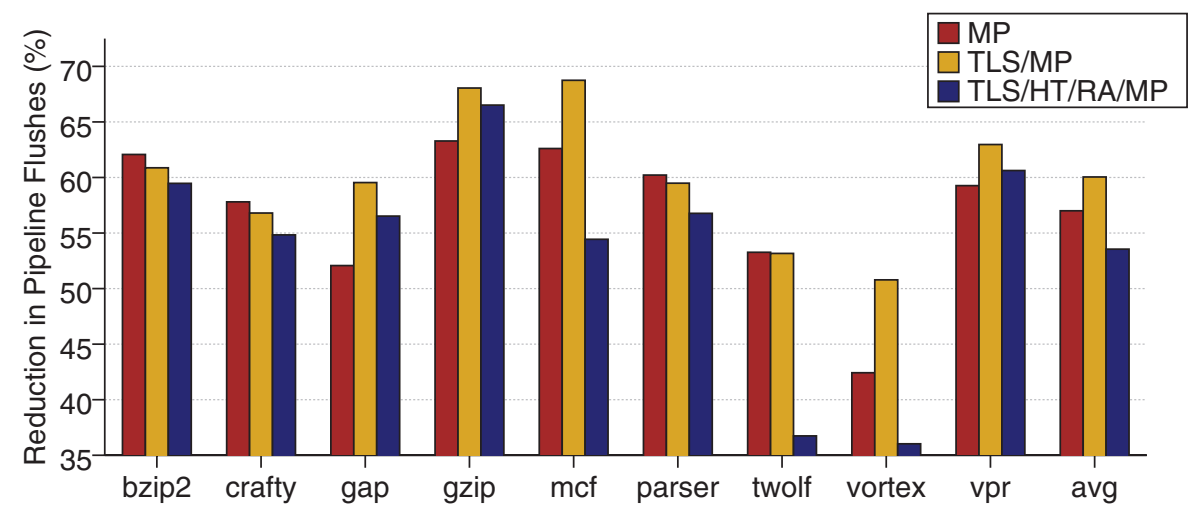

Fig. 10. Reduction in pipeline flushes for the MP system, and the combined TLS/MP and TLS/HT/RA/MP schemes.

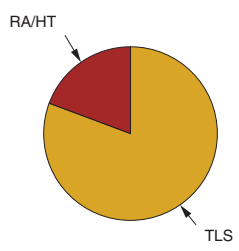

(a)

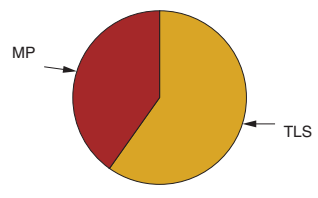

(b)

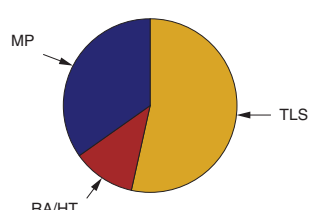

(c)

Fig. 11. Percentage of threads in each execution mode for (a) the TLS/HT/RA, (b) the TLS/MP and (c) the TLS/HT/RA/MP mixed execution models.

TLS/MP and TLS/HT/RA/MP models. Although some of the benefits of MP are not as significant as they were for the TLS/MP model, they are still quite substantial. This interesting interplay between prefetching and reduction in pipeline flushes, reveals the versatility of the mixed execution models. In fact the mixed models can naturally adapt to the different type of workloads at hand, since more misses in the L2 cache will trigger more prefetching whereas more mispredictions will trigger more MP execution.

\subsection{Interplay of the Execution Models}

In the previous sections, it has been shown that the major benefits of the mixed execution models come in the form of improved ILP. This improvement comes in terms of a reduction in the number of misses in the L2 cache and a reduction of the number of pipeline flushes due to branch mispredictions. This section tries to quantify the interplay of these different execution models.

Figure 11 presents the fraction of retired instructions that amount to each of the execution models. In order to represent the average behavior, we took the geometric mean of the percentages for each of the benchmarks.In Figure 11(a), which corresponds to the TLS/HT/RA mode, it can be seen that only $20 \%$ of the threads correspond to PTs. This is reasonable since most of the applications used for this study have a small amount of misses in the L2 cache, and because TLS threads have always priority over the PTs. For the TLS/MP model (Figure 11(b)), the story is somewhat different. Around $35 \%$ of the threads are now MP threads, mainly because the confidence estimator is biased to classifying branches as hard-to-predict. At the same time, the mapping policy for the TLS threads ensured that MP execution could be triggered most of the time. 


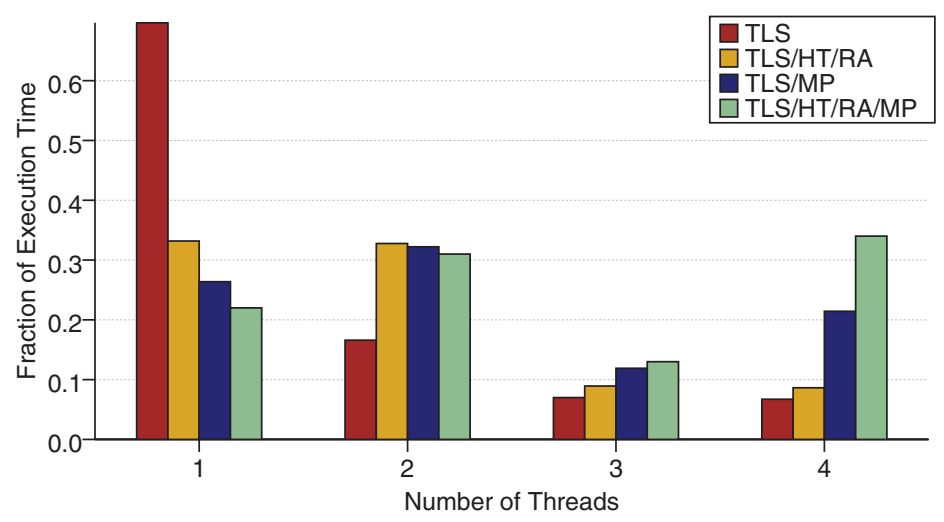

Fig. 12. Average distribution of number of concurrently running threads for TLS and the combined TLS/HT/RA, TLS/MP and TLS/HT/RA/MP schemes.

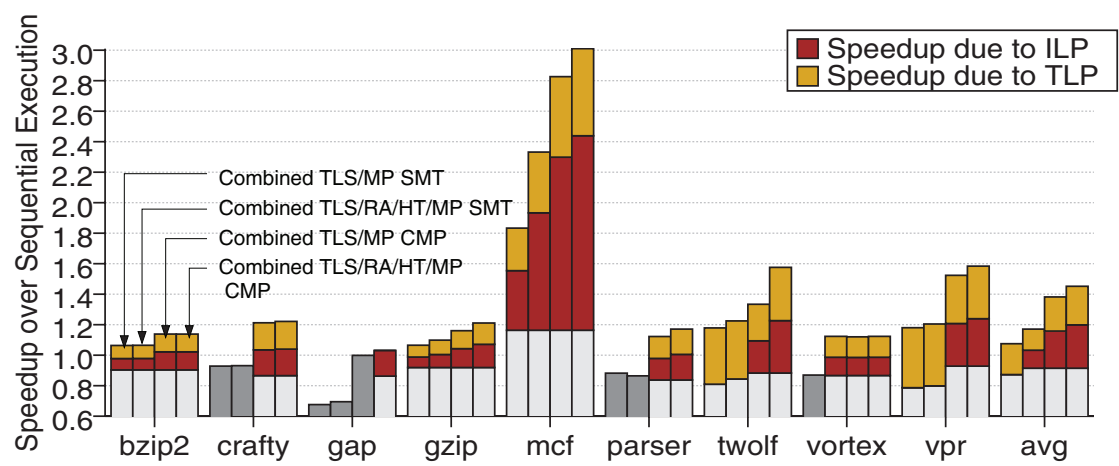

Fig. 13. Speedup over sequential execution for different mapping policies for the TLS/MP and TLS/HT/RA/MP schemes.

As Figure 11(c) reveals, the interplay for the TLS/HT/RA/MP model follows the same trends. The fraction of threads that correspond to TLS now drops to 55\% and that of the MP ones to $31 \%$. Note however, that this does not mean that there are fewer TLS threads, only that their proportion becomes smaller.

Figure 12 depicts the average number of concurrently running threads we have for each of the different mixed execution models and for base TLS. As it can be seen, under TLS usually there is only one thread executing and for a small fraction of the total time, two threads execute in parallel. As expected, for the combined models, the time spent executing only one thread is far smaller. The TLS/HT/RA/MP model as expected is the one that has in a significant fraction of the time (35\%) four or more threads running.

\subsection{Impact of Mapping Policies}

As previously mentioned, in order to accommodate MP execution, multiple-context support for each of the cores is required. This means that newly generated threads can be mapped either to the contexts available within the core or to a new core. Throughout this article, we have chosen the later for all threads except the MP ones. The reasoning was that in this way, we could spawn MP threads fast enough so as to benefit from them the most. Figure 13 compares the performance of the two alternatives for TLS/MP and TLS/HT/RA/MP. For the TLS/HT/RA model, it does not make much of a difference, and 


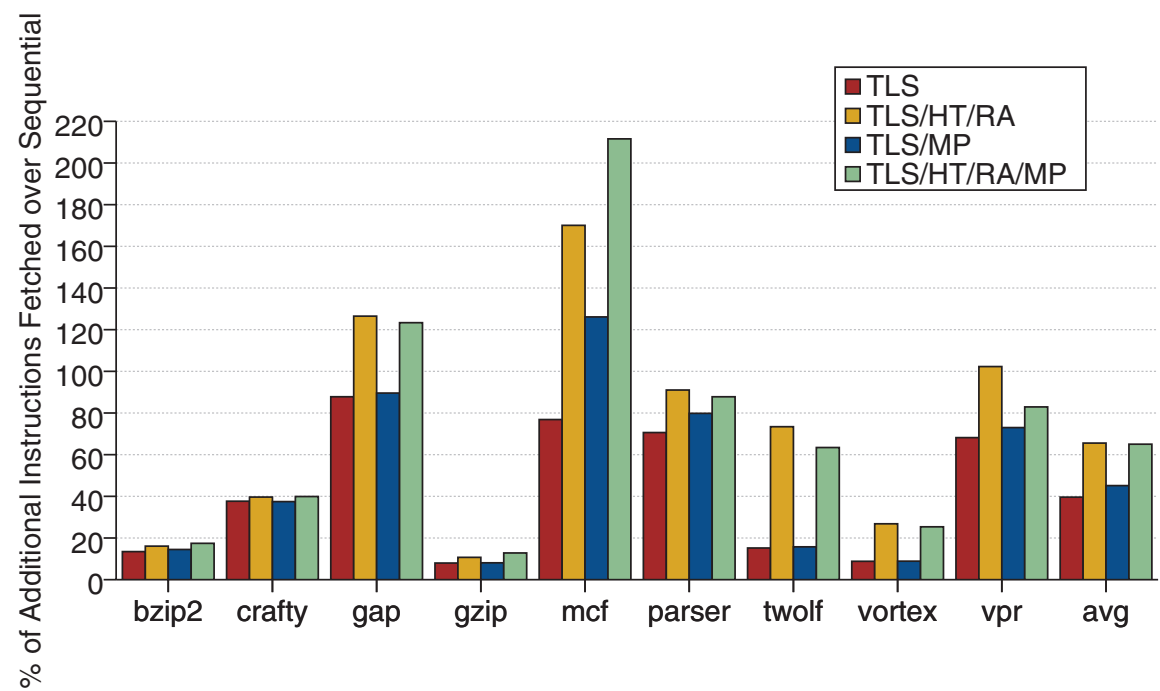

Fig. 14. Number of additional instructions executed over the baseline sequential system for all the different execution models.

for this case we do not present numbers for it. With $S M T$ we tag the cases where we try to map in a free processor context first, while with $C M P$ we tag our mapping policy.

For the TLS/MP, most of the benefit is negated in the SMT policy by the slow spawning of the MP threads. In fact as the graph reveals, almost all of the ILP benefit is removed on average. Similar results are shown for the TLS/HT/RA/MP model. Although under this model, there is still some ILP that is extracted, this is mostly ILP due to prefetching from the PTs. However, this is only a small fraction of the ILP that can be extracted, a fact that results in significant performance loss. These results demonstrate the importance of carefully selecting mapping policies that are appropriate for the execution models that are combined.

\subsection{Power Implications}

One important aspect of the speculative models analyzed so far is that the performance benefits come at the expense of executing additional instructions over the sequential case. Figure 14 depicts the additional instructions fetched under TLS and the combined execution models. As expected, the speculative execution models, including base TLS, execute a significant amount of additional instructions. Assuming a linear relationship between activity and consumed energy, the amount of energy increases by $62 \%$ over the sequential core for the TLS/HT/RA/MP model. Note that MP is more energy efficient than RA/HT which requires more instructions than any of the other models (with the exception of $m c f$ ). Interestingly, the TLS/HT/RA/MP model that combines MP and RA/HT on top of TLS and its additive performance benefits do not translate to additive instruction count.

Since the additional energy comes from executing more instructions, it has to be noted that the system will still operate under the thermal envelope for which it was designed. In fact, all the proposed models are a somewhat related to the Turbo boost technology that was recently introduced by Intel [2008]. Under that scheme, the frequency of one core can be increased in order to achieve performance benefits when other cores are idle. Arguably, for straight line code, Turbo Boost is more energy efficient than the combined execution models. However, for applications with limited ILP like the ones studied in this article, increasing the frequency will have only minimal performance 
benefits while increasing the energy consumed significantly. A thorough discussion of Turbo Boost with speculative execution models can be found in Ioannou and Cintra [2011].

\section{RELATED WORK}

\subsection{Mixed Execution Models}

Concurrently with our work [Xekalakis et al. 2009; Xekalakis and Cintra 2010], Sun announced its Rock processor [Chaudhry et al. 2009]. The Rock processor supports transactional memory and it uses the same hardware structures to also perform prefetching by means of scouting threads. Although the execution models are different, the basic idea of re-using the hardware support in order to allow multiple speculative execution models is the same. On a similar note, Nikas et al. [2009] combine HTs with transactional memory and shows that it can greatly improve performance when trying to parallelize Dijkstra's algorithm. Porter et al. [2009] combine transactional memory with TLS, leveraging upon the similarities of these two execution models. Recently Garg et al. [2011] proposed to exploit speculative parallelization, but only for the lookahead thread.

\subsection{Combined ILP/TLP Benefits}

Warg [2005] showed in a limit study that it might be beneficial to continue running some threads that are predicted to squash, so as to do prefetching. However, the benefits shown there were minimal if one considers the simplifications in the simulation infrastructure used. In general, deferring the squashes so as to prefetch is not beneficial because we negate the TLP benefits. Benefits due to prefetching were also reported for TLS systems in [Renau et al. 2005]. In our work we show that by employing a combined speculative multithreading approach, one can obtain further benefits from prefetching.

\subsection{Thread Level Speculative Systems}

Thread level speculation has been previously proposed [Hammond et al. 1998; Krishnan and Torrellas 1998; Marcuello and González 1999; Sohi et al. 1995; Steffan and Mowry 1998] as a means to provide some degree of parallelism in the presence of data dependences. The vast majority of prior work on TLS systems has focused on architectural features directly related to the TLS support, such as protocols for multiversioned caches and data dependence violation detection. All these are orthogonal to our work. In particular, we use the system in Renau et at [2005] as our baseline.

\subsection{Helper Threads}

It has been previously proposed to use small helper threads on otherwise idle hardware resources [Chappell et al. 1999; Collins et al. 2001; Sundaramoorthy et al. 2000; Zilles and Sohi 2001]. There, helper threads usually try to resolve highly unpredictable branches and cache misses that the main thread would have to stall upon otherwise. Because helper threads try to improve the ILP of the main thread, they fail to procure any significant benefits in applications where the out-of-order engine is able to extract most of the ILP. As we showed in previous sections, in these cases we can achieve some additional improvements by trying to extract TLP as well.

\subsection{Runahead Execution}

Runahead execution [Barnes et al. 2003; Dundas and Mudge 1997; Mutlu et al. 2003] is a scheme that generates accurate data prefetches by speculatively executing past a long latency cache miss, when the processor would otherwise be stalled. Runahead 
execution is similar to the helper thread schemes, although instead of using different hardware resources, it uses otherwise idle processor cycles to perform prefetching. Additionally, runahead execution does not rely on the programmer to manually extract the prefetching slices. Two more recent proposals, Checkpointed Early Load Retirement [Kirman et al. 2005] and CAVA [Ceze et al. 2006], build on Mutlu et al.[2003] by adding value prediction. In contrast with runahead execution, correctly predicting the value of a missing load eliminates the need to rollback to a checkpoint when the load returns. Ceze et al. [2006] showed, however, that most of the benefits from this scheme do not come from negating the roll-backs, but rather from the fact that by value predicting, prefetches are more accurate. As with the helper thread case, runahead execution tries to improve the ILP of memory bound (mainly) applications by prefetching. Our scheme is able to achieve speedups even for applications that are not memory bound. Additionally, because we are able to perform deeper prefetching than runahead execution, even for memory bound applications we are able to achieve better results.

\subsection{Multipath Execution}

A significant amount of work has been done in the area of systems able to execute multiple control flow paths [Ahuja et al. 1998; Heil et al. 1996; Klauser et al. 1998; Kim et al. 2006]. All these studies have shown that being able to follow multiple paths is always beneficial. In fact, some of these proposals advocate following not only one but multiple control flow paths simultaneously. Recently, Kim et al. [2006] showed that combining compiler information with runtime behavior is the best approach to follow, both in terms of speedup and energy efficiency. As future work we wish to explore mixing the TLS execution model with a flavor of the Diverge-Merge scheme. All these studies are based on SMT (or SMT-like) systems and assume fast register copying. Chidester et al. [2003] employ a form of slipstream execution to allow multiple-path execution on tightly coupled multicores. Unfortunately, this proposal assumes very tight coupling of the cores, through a communication queue, and it does not scale well when the delay of the queue increases.

\section{CONCLUSIONS}

This article seeks to enhance TLS functionality by combining it with other speculative multithreaded execution models. The main idea is that TLS already requires extensive hardware support, which when slightly augmented can accommodate other speculative multithreaded techniques. Recognizing that for different applications, or even program phases, the application bottlenecks may be different, it is reasonable to assume that the more versatile a system is, the more efficiently it will be able to execute the given program.

Three execution models are chosen so as to complement TLS, namely RunAhead execution, Helper Threads and MultiPath execution. This new mixed execution model, greatly outperforms any of the simpler models alone mainly because it is able to extract ILP either by removing misses to the L2 cache or reducing the pipeline flushes due to branch mispredictions. At the same time, it does so while also extracting as much TLP as the base TLS system is able to uncover. Experimental results show that this mixed execution model is better than the best simple model by as much as $82 \%$ with an average of $14 \%$. This in turn results in an average speedup of $45 \%$ over sequential execution.

\section{REFERENCES}

Ahuja, P., Skadron, K., Martonosi, M., and Clark, D. 1998. Multipath execution: Opportunities and limits. In Proceedings of the International Conference on Supercomputing, 101-108. 
Aragon, J. L., GonzÁlez ,J., GarcA, J. M., AND GonzÁLez, A. 2001. Confidence estimation for branch prediction reversal. In Proceedings of the International Conference on High Performance Computing, 213-224.

Barnes, R., Nystrom, E., Sias, J., Patel, S., Navarro, N., and Hwu, W. M. 2003. Beating in-order stalls with "flea-ficker" two-pass pipelining. In Proceedings of the International Symposium on Microarchitecture. 387-398.

Ceze, L., Strauss, K., Tuck, J., Renau, J., and Torrellas, J. 2006. CAVA: Using checkpoint-assisted value prediction to hide L2 misses. ACM Trans. Architecture Code Optim. 3, 2, 182-208.

Chappell, R. S., Stark, J., Kim, S. P., Reinhardt, S. K., And Patt, Y. N. 1999. Simultaneous subordinate microthreading (SSMT). In Proceedings of the International Symposium on Computer Architecture. 186-195.

Chappell, R. S., Tseng, F., Patt, Y. N., and Yoaz, A. 2002. Difficult-path branch prediction using subordinate microthreads. In Proceedings of the International Symposium on Computer Architecture. 307-317.

Chaudhry, S., Cypher, R., Ekman, M., Karlsson, M., Landin, A., Yip, S., Zeffer, H., and Tremblay, M. 2009. Simultaneous speculative threading: A novel pipeline architecture implemented in Sun's ROCK Processor. In Proceedings of the International Symposium on Computer Architecture. 484-495.

Chidester, M. C., George, A. D., And Radlinski, M. A. 2003. Multiple-path execution for chip multiprocessors. J. Syst. Archit. 33-52.

Collins, J. D., Wang, H., Tullsen, D. M., Hughes, C., Lee, Y.-F., Lavery, D., and Shen, J. P. 2001. Speculative precomputation: Long-range prefetching of delinquent loads. In Proceedings of the International Symposium on Computer Architecture. 14-25.

Dundas, J. AND MudGE, T. 1997. Improving data cache performance by pre-executing instructions under a cache miss. In Proceedings of the International Conference on Supercomputing. 68-75.

GARg, A., PARIhar, R., AND Huang, M. C. 2011. Speculative parallelization in decoupled look-ahead. In Proceedings of the International Conference on Parallel Architectures and Compilation Techniques. 413-423.

Grunwald, D., Klauser, A., Manne, S., And Pleszkun, A. 1998. Confidence estimation for speculation control. In Proceedings of the International Symposium on Computer Architecture. 122-131.

Hammond, L., Wiley, M., AND Olukotun, K. 1998. Data speculation support for a chip multiprocessor. In Proceedings of the International Conference on Architectural Support for Programming Languages and Operating Systems. 58-69.

Heil, T. ANd Smith, J. E. 1996. Selective dual path execution. Tech. Rep., Department of Electrical and Computer Engineering, University of Wisconsin-Madison.

INTEL CORP. Intel turbo boost technology in intel core microarchitecture (nehalem) based processors. http://download.intel.com/design/processor/applnots/320354.pdf.

IOANNOU, N. AND CinTRA, M. 2011.Complementing user-level coarse-grain parallelism with implicit speculative parallelism. In Proceedings of the International Symposium on Microarchitecture. 284-295.

Kim, H., JoAo, J. A., Mutlu, O., AND PATt, Y. N. 2006. Diverge-Merge Processor (DMP): Dynamic predicated execution of complex control-flow graphs based on frequently executed paths. In Proceedings of the International Symposium on Microarchitecture. 53-64.

Kirman, N., Kirman, M., Chaudhuri, M., and Martínez, J. F. 2005. Checkpointed early load retirement. In Proceedings of the International Symposium on High-Performance Computer Architecture. 16-27.

Klauser, A., Paithankar, A., And Grunwald, D. 1998. Selective eager execution on the polypath architecture In Proceedings of the International Symposium on Computer Architecture. 250-259.

KRISHNAN, V. AND TORRELLAS, J. 1998. Hardware and software support for speculative execution of sequential binaries on a chip-multiprocessor. In Proceedings of the International Conference on Supercomputing, 85-92.

Liu, W., Tuck, J., Ceze, L., Ahn, W., Strauss, K., Renau, J., and Torrellas, J. 2006. POSH: A TLS compiler that exploits program structure. In Proceedings of the Symposium on Principles and Practice of Parallel Programming. 158-167.

Marcuello, P. And González, A. 1999. Clustered speculative multithreaded processors. In Proceedings of the International Conference on Supercomputing. 365-372.

Mutlu, O., Stark, J., Wilkerson, C., and Patt, Y. N. 2003. Runahead execution: An alternative to very large instruction windows. In Proceedings of the International Symposium on High-Performance Computer Architecture. 129-140.

Nikas, K., Anastopoulos, N., Goumas, G., And KoziRis, N. 2009. Employing transactional memory and helper threads to speed up Dijkstra's algorithm. In Proceedings of the International Conference on Parallel Processing, 388-395. 
Porter, L. Choi, B., And Tullsen, D. M. 2009. Mapping out a path from hardware transactional memory to speculative multithreading. In Proceedings of the International Conference on Parallel Architectures and Compilation Techniques. 313-324.

RENAU, J. SESC simulator. http://sesc.sourceforge.net.

Renau, J., Tuck, J., Liu, W., Ceze, L., Strauss, K., and Torrellas, J. 2005. Tasking with out-of-order spawn in TLS chip multiprocessors: Microarchitecture and compilation. In Proceedings of the International Conference on Supercomputing. 179-188.

SEznec, A. 2005. Analysis of the OGEHL predictor. In Proceedings of the International Symposium on Computer Architecture, 394-405.

Sohi, G. S., Breach, S. E., And Vijaykumar, T. N. 1995. Multiscalar processors. In Proceedings of the International Symposium on Computer Architecture. 414-425.

Steffan, J. G. AND Mowry, T. C. 1998. The potential for using thread-level data speculation to facilitate automatic parallelization. In Proceedings of the International Symposium on High-Performance Computer Architecture. 2-13.

Sundaramoorthy, K., Purser, Z., And Rotenberg, E. 2000. Slipstream processors: Improving both performance and fault tolerance. In Proceedings of the International Conference on Architectural Support for Programming Languages and Operating Systems. 257-268.

Tarjan, D., Thoziyoor, S., And Jouppi, N. P. 2006. Cacti 4.0. Tech. Rep., Compaq Western Research Lab.

Tullsen, D. M., Eggers, S. J., Emer, J. S., Levy, H. M., Lo, J. L., And Stamm, R. L. 1996. Exploiting choice: Instruction fetch and issue on an implementable simultaneous multithreading processor. In Proceedings of the International Symposium on Computer Architecture. 191-202.

WARG, F. 2005. Reducing misspeculation overhead for module-level speculative execution. In Proceedings of the International Conference on Computing Frontiers. 289-298.

XeKalakis, P. And Cintra, M. 2010. Handling branches in TLS systems with multi-path execution. In Proceedings of the International Symposium on High-Performance Computer Architecture. 367-378.

Xekalakis, P., Ioannou, N., and Cintra, M. 2009. Combining thread level speculation, helper threads, and runahead execution. In Proceedings of the International Conference on Supercomputing. 410-420.

Zilles, C. AND Sohi, G. 2001. Execution-based prediction using speculative slices. In Proceedings of the International Symposium on Computer Architecture. 2-13.

Received December 2010; revised July 2011; accepted March 2012 